\title{
A Systematic Search for Short-term Variability of EGRET Sources
}

\author{
P. M. Wallace', D. L. Bertsch ${ }^{2}$, S. D. Bloom ${ }^{3}$, N. J. Griffis ${ }^{1}$, S. D. Hunter ${ }^{2}$, \\ D. A. Kniffen ${ }^{4}$, D. J. Thompson ${ }^{2}$ \\ ${ }^{1}$ Department of Physics and Astronomy, Berry College, Mt. Berry, GA 30149 \\ ${ }^{2}$ NASA/Goddard Space Flight Center, Greenbelt, MD 20771 \\ ${ }^{3} I P A C, J P L / C a l t e c h, M S 100-22$, Pasadena, CA 91125 \\ ${ }^{4}$ Department of Physics and Astronomy, Hampden-Sydney College, Hampden-Sydney, VA 23943 and \\ NASA HQ, Washington, DC 20546
}

\begin{abstract}
The 3rd EGRET Catalog contains 170 unidentified high-energy ( $\mathrm{E}>100 \mathrm{MeV}$ ) gamma-ray sources, and there is great interest in the nature of these sources. One means of determining sources class is the study of flux variability on time scales of days; pulsars are believed to be stable on these scales while blazars are known to be highly variable. In addition, previous work has led to the discovery of 2CG 135+01 and GRO J1838-04, candidates for a new high-energy gamma-ray source class. These sources display transient behavior but cannot be associated with any known blazars. These considerations have led us to conduct a systematic search for short-term variability in EGRET data, covering all viewing periods through cycle 4. Three unidentified sources show some evidence of variability on short time scales; the source displaying the most convincing variability, 3EG J2006-2321, is not easily identified as a blazar.
\end{abstract}

\section{INTRODUCTION}

There are 271 sources listed in the 3rd EGRET Catalog of High-energy Gamma-ray Sources'. Besides one solar flare, the Large Magellanic Cloud, and a possible association with a radio galaxy (Cen A), the identified sources are distributed among two established classes of high-energy gamma rays: pulsars and radio-loud blazars. Pulsars are believed to not vary in gamma-ray output over time scales of one or two days, while blazars are known to be highly variable. While many instances of blazar flares have been reported, no comprehensive survey of EGRET data has been performed. It is the purpose of this study to conduct a systematic search for short-term variability in EGRET data from cycles 1-4. This paper focuses on the unidentified 3EG sources.

\section{DATA \& ANALYSIS}

All unidentified 3EG sources are examined across all viewing periods (VP's) for evidence of variability. The VP's are broken down into one-day intervals and intensity

CP510, The Fifth Compton Symposium, edited by M. L. McConnell and J. M. Ryan (C) 2000 American Institute of Physics 1-56396-932-7/00/\$17.00 
maps are generated for each day. With such short intervals, the statistics are extremely limiting; therefore this study is sensitive to only the strongest changes in gamma-ray output. Only those light curves with at least one $4 \sigma$ one-day detection are considered for close analysis.

The remaining light curves are analyzed using the variability index $V$. If $Q$ is the probability of obtaining a value of $\chi^{2}$ equal to or greater than the empirical $\chi^{2}$ from an intrinsically nonvariable source, then $V \equiv-\log Q$. All curves with $V \leq 1.0$ are considered to be not variable. The curves are also inspected for evidence of flaring; those that display such evidence are modeled by Monte Carlo methods in order to determine the probability of finding such a flare from an intrinsically stable source. Three unidentified 3EG sources displayed $V \geq 1.0$ and/or evidence of flaring. They are discussed below.

\section{EG J1410-6151}

During the first four days of VP 14.0, the flux of 3EG J1410-6151 fell from $(5.4 \pm 1.5) \times 10^{-6}$ photons $\mathrm{cm}^{-2} \mathrm{~s}^{-1}$ to below EGRET's sensitivity where it remained for the rest of the 14-day period; this is suggestive of flaring behavior. (See Figure 1.) Monte Carlo simulation gives a probability of 0.0007 that the fluctuation found in this VP is produced by a nonvariable source. It has been suggested ${ }^{2}$ that $3 E G$ J1410-6103 $(l=312.18, b=-0.35)$ is associated with SNR G312.4-0.4, which falls just outside the $68 \%$ error contour. It should be noted that although this VP occurred early in EGRET's life when its sensitivity was high, the source is $27^{\circ}$ off-axis.

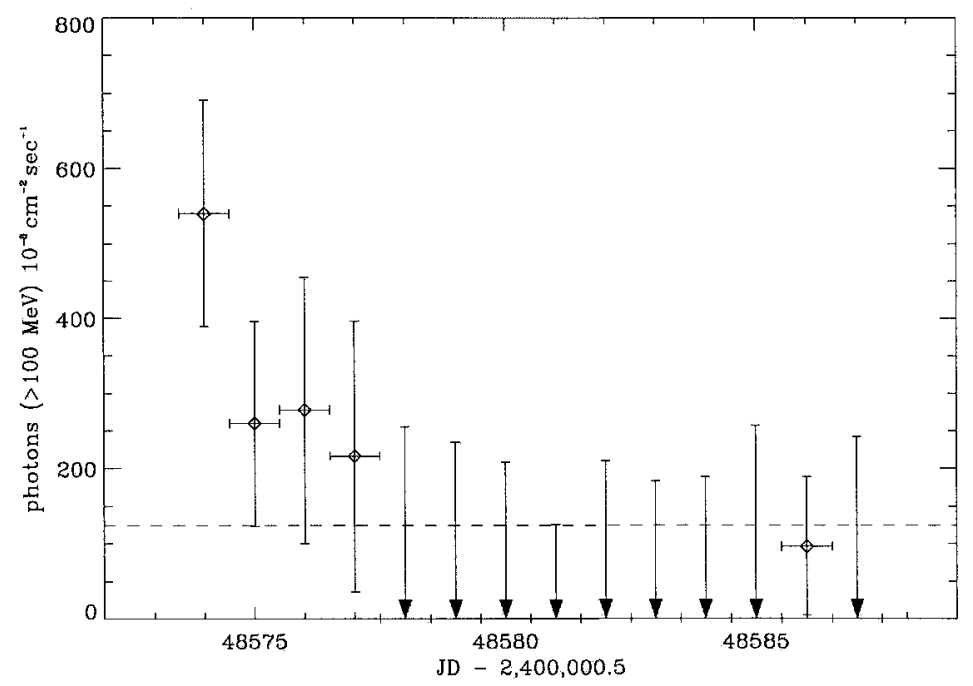

FIGURE 1. Light curve of 3EG J1410-6151 from VP 14.0. $V=1.47$. 


\section{EG J1746-2851}

As this source is unidentified, strong, and coincident with the Galactic Center, it has been studied in some detail ${ }^{3}$. However, until now its short-term variability has not been examined, and there is some evidence of variability in VP's 16.0 and 429.0. 3EG J1746-2851 sits in the most densely-packed region of the high-energy gamma-ray sky; there are ten sources listed in the 3rd EGRET Catalog within $10^{\circ}$ of the Galactic Center. Given the broad EGRET PSF, source confusion is a serious problem. However, while 3EG J1746-2861 appears to fluctuate during two different VP's, no other sources in confused regions display any evidence of short-term variability.

The light curve of 3EG J1746-2851 during VP 16.0 is shown in Figure 2. The three strongest one-day detections fall on days 7-9 of the two-week VP, during which the aspect was $20^{\circ}$. The peak detection has a significance of $4.3 \sigma$ and is flanked by detections of $3.9 \sigma$ and $3.1 \sigma$. The variability index is 2.09 , corresponding to a probability of 0.008 that these data are consistent with a nonvariable source. Monte Carlo analysis is more restrictive. This source and the seven others within a $7^{\circ}$ radius were modeled and there is a probability of 0.0004 that a three-day fluctuation of this or greater significance will occur in a 14-day period given intrinsically nonvariable sources.

3EG J1746-2851 also shows evidence of variability in VP 429.0. During this pointing the aspect is only $6^{\circ}$ and $V=3.0$. The peak flux is $(6.4 \pm 1.7) \times 10^{-6}$ photons $\mathrm{cm}^{-2} \mathrm{~s}^{-1}$ and on two days the source is not detected at all, but there is no evidence of flaring.

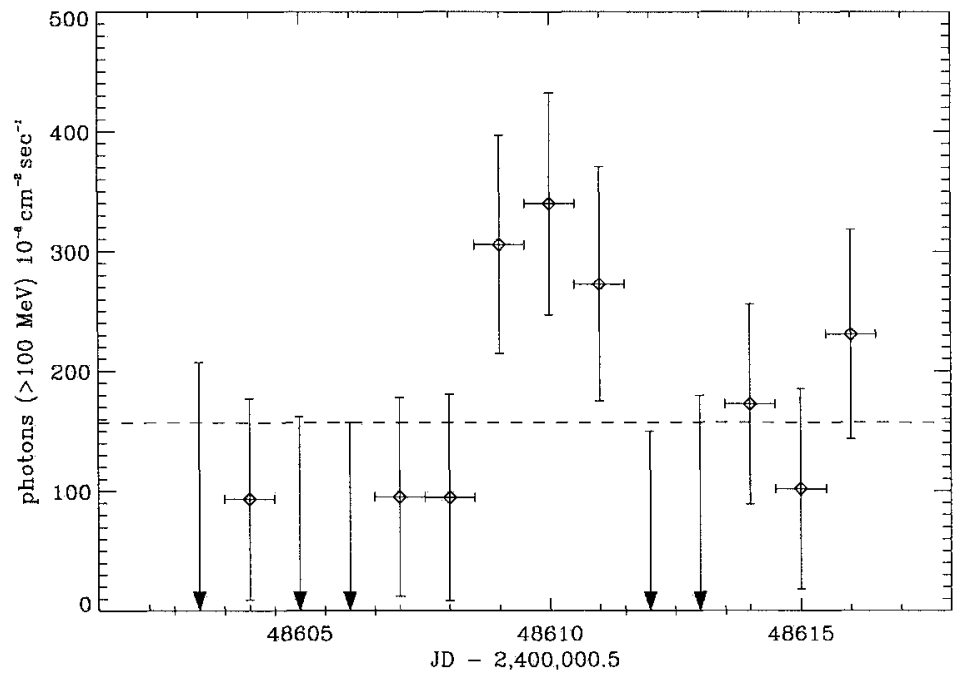

FIGURE 2. Light curve of 3EG J1746-2851 from VP 16.0. $V=2.09$. 


\section{EG J2006-2321}

This source shows strong variability in VP 13.1, during which it was $13^{\circ}$ from the instrument axis. The light curve shows evidence of flaring and is shown in Figure 3. The variability index for this curve is 3.18; the Monte Carlo probability that the source is nonvariable is 0.0006 . 3EG J2006-2321 is well-isolated and lies $26^{\circ}$ off the Galactic Plane, free of the bright galactic diffuse radiation; thus the claim of variability is strengthened.

The combination of large $|b|$ and flaring behavior suggests an association with the blazar class of AGN. However, all of the 66 3EG sources identified as AGN are associated with loud spectrally flat radio sources; for 3EG 2006-2321 no such association can be easily made. The best candidate is the radio source PMN J2005$2310\left(260 \mathrm{mJy}\right.$ at $4.85 \mathrm{GHz}, \alpha_{\mathrm{r}}$ not known), for which the probability of association ${ }^{4}$ with 3EG J2006-2321 is only 0.015 . If this source is of extragalactic origin, then it is unlike other EGRET AGN; of the 10 AGN with peak flux above $10^{-6}$ photons $\mathrm{cm}^{-2} \mathrm{~s}^{-1}$, none are weaker than $1.0 \mathrm{Jy}$ at $4.85 \mathrm{GHz}$.

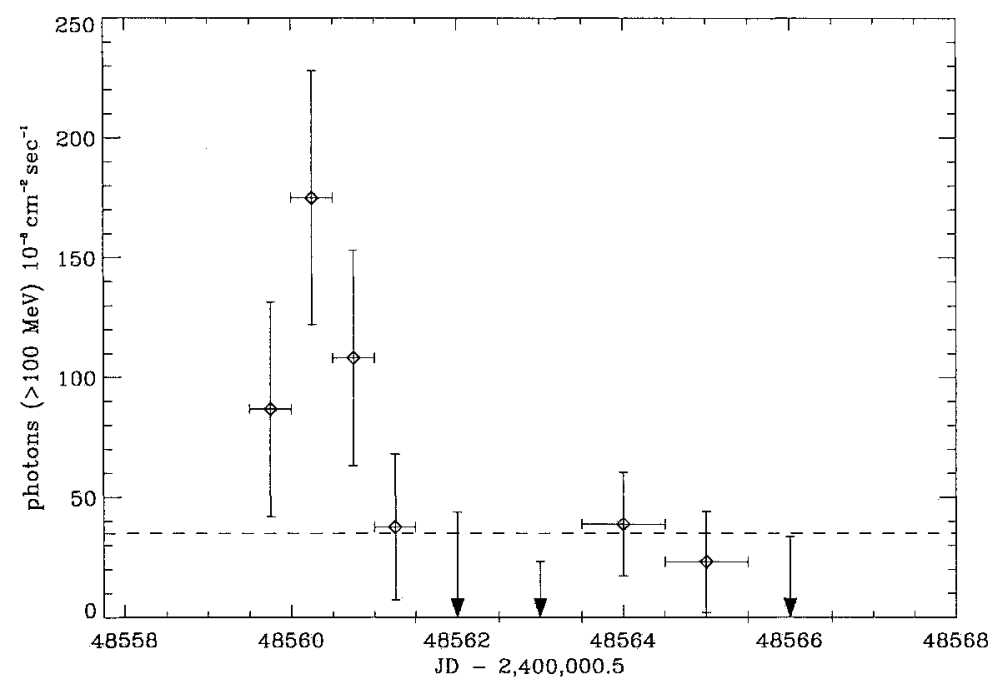

FIGURE 3. Light curve of 3EG J2006-2321 from VP 13.1. $V=3.18$. The first 4 points represent $12-$ hour integration times; the final 5 represent 24-hour integration times.

Recently, two other sources have been found to share this combination of variability, peak flux above $10^{-6}$ photons $\mathrm{cm}^{-2} \mathrm{~s}^{-1}$, and lack of easy association with a radio-loud spectrally flat counterpart: 2CG $135+01$ and GRO J1838-04, . An association of 2CG 135+01 (3EG J0241+6103) with the radio source GT $0236+610$ has been suggested but not confirmed; GT $0236+610$ itself is associated with the massive binary system LS $I+61^{\circ} 303$. To date there are not plausible counterparts, galactic or extragalactic, for GRO J1838-04 (3EG J1837-0423). 3EG J2006-2321, 
along with these two sources, may be representative of a new class of high-energy gamma-ray emitters. However, there are some differences among these three sources; unlike 3EG J2006-2321, the other two sources are very close to the Galactic Plane. Also, while 2 CG $135+01$ is found by the present study to be variable on short time scales, GRO 1838-04 is not; it is a very bright but steady source in VP 423.0.

Further study of possible association of this source with PMN J2005-2310 is underway.

\section{CONCLUSION}

The survey of EGRET data from cycles 1-4 finds three unidentified sources that display some evidence of short-term variability; large statistical errors ensure that we detect only the strongest variations. Of these three, only 3EG J2006-2321 is strongly variable. If this source is an AGN, its radio characteristics are unlike those of other bright (peak flux $>10^{-6}$ photons $\mathrm{cm}^{-2} \mathrm{~s}^{-1}$ ) EGRET blazars. If it is not extragalactic in origin, it may, with 2CG 135+01 and GRO J1838-04, represent a new class of highenergy gamma-ray emitters. Study of this source continues.

\section{REFERENCES}

1. Hartman, R. C. et al. 1999 ApJS, 123, 79

2. Sturner. S. J. \& Dermer, C. D. 1995, A\&A, 293, L17

3. Mayer-Hasselwander, H. A. et al. 1998, A\&A 335,161

4. Mattox, J. R. 1999, these proceedings

5 Kniffen, D. A. et al. 1997, ApJ, 486, 126

6. Tavani, M. et al. 1998, ApJ, 497, L89 